Research Paper

\title{
Prevalence and Genotyping of Cryptosporidium parvum in Gastrointestinal Cancer Patients
}

\author{
Nan Zhang ${ }^{1 *}$, Xiuyan $\mathrm{Yu}^{2 *}$, Hongbo Zhang ${ }^{3 *}$, Lianying Cui ${ }^{2}$, Xiaoou $\mathrm{Li}^{2}$, Xiaowei Zhang ${ }^{2}$, Pengtao Gong ${ }^{3}$, \\ Jianhua $\mathrm{Li}^{3}$, Ziyi $\mathrm{Li}^{1}$, Xiaocen Wang ${ }^{3}$, Xin $\mathrm{Li}^{3}$, Ting $\mathrm{Li}^{2}$, Xiaofeng $\mathrm{Liu}^{2}$, Yanhui $\mathrm{Yu}^{\star 凶}$ and Xichen Zhang ${ }^{3 凶}$ \\ 1. The First Hospital, Key Laboratory of Zoonosis Research by Ministry of Education, Institute of Zoonosis, Jilin University, Changchun 130021, China. \\ 2. Jilin Cancer Hospital, Changchun 130021, China. \\ 3. Key Laboratory of Zoonosis Research by Ministry of Education, Institute of Zoonosis, College of Veterinary Medicine, Jilin University, Changchun 130062, \\ China. \\ 4. Clinical Lab, The Second Hospital, Jilin University, Changchun 130021, China.
}

*These authors contributed equally to this work.

$\triangle$ Corresponding authors: Xichen Zhang, Key Laboratory of Zoonosis Research by Ministry of Education, Institute of Zoonosis, College of Veterinary Medicine, Jilin University, Changchun 130062, China. Email: xczhang@jlu.edu.cn and Yanhui Yu, Clinical Lab, The Second Hospital, Jilin University, Changchun 130021, China. Email: 1053408972@qq.com.

(1) The author(s). This is an open access article distributed under the terms of the Creative Commons Attribution License (https://creativecommons.org/licenses/by/4.0/). See http://ivyspring.com/terms for full terms and conditions.

Received: 2019.11.23; Accepted: 2020.02.03; Published: 2020.03.05

\begin{abstract}
Gastrointestinal cancers are the most commonly occurring malignancies which contributing to over $1 / 5$ of cancer incidences worldwide. Increasing evidences have shown that Cryptosporidium spp., an apicomplexan protozoan, is highly associated with gastrointestinal cancers. However, the prevalence of Cryptosporidium spp. infections among gastrointestinal cancer patients in China has not been estimated yet. We here performed a case-control study to evaluate the occurrences of Cryptosporidium spp. in patients with digestive malignancies before chemotherapy and in control population. Nested PCR amplifying $18 \mathrm{~S}$ rRNA gene was used to detect the presence of Cryptosporidium spp. in each fecal sample. The results herein confirmed the correlation of Cryptosporidium spp. infection with colorectal and liver cancers, while first identified the high frequencies of Cryptosporidium spp. in esophageal cancer and small intestine cancer. The infection rates of Cryptosporidium spp. in colorectal, esophageal, liver and small intestine cancers were $17.24 \%(20 / 116, P<0.001), 6.25 \%(1 / 16, P=0.029), 14.29 \%(1 / 7, P<0.001)$ and $40 \%$ $(2 / 5, P<0.001)$, respectively. In addition, molecular characterization indicated that all the Cryptosporidium spp. obtained were Cryptosporidium parvum (C. parvum), and the I8S rRNA sequences were identical to the reference sequences isolated from cattle, suggesting potential zoonotic transmission. Furthermore, subtyping analyses revealed that IlaA15G2R1 and IlaA15G2R2 were the predominant subtypes in colorectal cancer, while IlaA13G2R2 subtype was first named and identified in colorectal and liver cancers. Taken together, for the first time, the prevalence of Cryptosporidium spp. infections in digestive cancer patients has been estimated among Chinese. Our results indicated that $C$. parvum were highly associated with gastrointestinal cancers, supporting that cryptosporidiosis could be a potential risk factor for these diseases.
\end{abstract}

Key words: C. parvum, Epidemiology, Colorectal cancer, Liver cancer, Esophageal cancer, Small intestine cancer

\section{Introduction}

Gastrointestinal cancers are the most commonly diagnosed cancers and the leading cause of cancer deaths with over $30 \%$ for mortality in 2018 [1,2]. Genetic or epigenetic defects are considered to be the causative reasons for a variety of digestive malignancies. Nevertheless, infectious agents, which contributed to about $20 \%$ of the cancer incidence worldwide, also cannot be ignored [3]. Thus far, a number of infectious agents have been identified as causative factors or contributors of various gastrointestinal cancers. The well-known infectious agents, Hepatitis $\mathrm{B}$ and $\mathrm{C}$ viruses; are the leading risk factors for liver cancer [4]. Helicobacter pylori infection is a major cause of gastric cancer [4]. In addition, some 
parasites, such as Opisthorchis viverrini and Clonorchis sinensis, are also considered to play a crucial role in digestive malignancies [3].

Cryptosporidium spp., belongs to apicomplexa protozoan, is a common and important zoonotic pathogen. It infects a wide range of hosts including humans and mainly inhabits the gastrointestinal and respiratory epithelium of vertebrates [5]. Cryptosporidium infection is a primary etiology of diarrhea in humans. Its secondary infection in AIDS (Acquired Immune Deficiency Syndrome), tumor and organ transplant patients is one of the major causes of death of these patients who have suppressed immune system [6,7]. So far, more than 30 species and 70 subtypes of Cryptosporidium have been identified [7,8]. Among them, Cryptosporidium hominis (C. hominis) and Cryptosporidium paroum (C. paroum) are the dominant pathogenic species responsible for most of the infection cases in humans [5]. Cryptosporidium is transmitted by fecal-oral route, mainly through contaminated food or water. It can also be transmitted to humans through direct contact with infected persons. In spite of the severe threat of Cryptosporidium infection to immunocompetent and immunocompromised individuals, there is still no ideal prevention and treatment method of cryptosporidiosis till now.

Cryptosporidium spp. infections have been suggested to be associated with several cancers, such as blood cancer, colorectal cancer and liver cancer [5]. As early as 1988, it has been suspected that Cryptosporidium spp. infection may be correlated with colon adenocarcinoma [9]. After that, the occurrences of Cryptosporidium spp. infections in colorectal cancer patients have been evaluated in different populations. In 2007, the first epidemiological study in Poland showed that 10 out of $55(18 \%)$ patients with diagnosed colorectal cancer had cryptosporidiosis, and the infection rate was even higher in those patients with diarrhea symptom $(43.5 \%, 10 / 55)$ [10]. Another two reports also conducted in Poland showed similar infection rates $(12.6 \%, 11 / 87$ and $13 \%$, 14/108) [11,12]. Recently, Osman M. et al. (2017) performed a systematic analysis among Lebanese patients which indicated that Cryptosporidium spp. infections were more prevalent in patients with colon adenocarcinoma $(21 \%, 15 / 72)$ than that in patients without digestive cancers but with digestive symptoms $(7 \%, 9 / 125)$ [13]. This study also identified C. hominis and C. parvum as the causative species of Cryptosporidium spp. Infections in colorectal cancer patients. In addition, a study in Saudi showed high frequencies of Cryptosporidium spp. in both colorectal cancer $(50 \%, 6 / 12)$ and liver cancer $(50 \%, 2 / 4)$ [14]. The high association between cryptosporidiosis and hepatocellular carcinoma $(32 \%, 16 / 32)$ was also suggested by another study in Egypt [15]. Nonetheless, the infection rates of Cryptosporidium spp. in other digestive cancers have not been determined yet.

So far, the occurrences of Cryptosporidium spp. in China have been evaluated among diarrheal outpatients, AIDS patients and children [16-20]. However, Cryptosporidium spp. infections in patients with gastrointestinal cancers have not been investigated in China. Thus, the aim of this study was to estimate the prevalence and characterize the species and genotypes/subtypes of Cryptosporidium spp. in gastrointestinal cancer patients in Jilin province, China.

\section{Materials and Methods}

\section{Ethics Statement}

Ethical approval of this study was obtained from the Ethical Committee of Jilin Cancer Hospital and the Second Hospital of Jilin University (Reference no.: 201712-047). The purpose of the study was orally explained to all the participants, and the fecal samples were collected with their approval.

\section{Study populations}

This study included 195 patients with diagnosed gastrointestinal cancers and without chemotherapy at Jilin Cancer Hospital and the Second Hospital of Jilin University during January-July, 2018. The cancer cases were determined based on imaging (B ultrasound; computed tomography, CT; nuclear magnetic resonance, NMR) and pathological diagnosis (gold standard). This group consisted of 125 males and 70 females with the average age of 59 years. We also recruited a control group of 141 individuals without diagnosis and history of cancer. The control population was confirmed by health examination in the hospital. This group included 70 males and 71 females and has an average age of 60 years. The detailed information of cancer patients and control population were shown in Table S1 and S2. We have previously used the same samples to detect the presence of Pentatrichomonas hominis in gastrointestinal cancer patients [21].

\section{Sample collection and DNA extraction}

The fresh fecal samples from 336 individuals were collected in empty containers and were cooled with ice during transport to the lab. All of the samples were stored at $-20^{\circ} \mathrm{C}$ until DNA extraction. In order to avoid the risk of cross-contamination, standard procedures for cleaning workspaces and instruments were applied. In addition, internal reference controls were set up at the same time. 
Genomic DNA was extracted from each fecal sample using the Fecal DNA Rapid Extraction Kit (TIANGEN, Beijing, China) according to the manufacturer's instructions. The purified DNA samples were stored at $-20^{\circ} \mathrm{C}$ until PCR analysis.

\section{PCR amplification}

The presence of Cryptosporidium spp. in the fecal samples were detected via nested PCR amplifying $\sim 587 \mathrm{bp}$ fragment of the $18 \mathrm{~S}$ rRNA gene. The primer sequences and PCR conditions were used as previously described [22]. The positive samples were further characterized for the subtypes of $C$. paroum by nested PCR amplifying gp60 sequences as previously described [23]. DNA extracted from C. parvum (IIa A15G2R1) was used as positive control, while $\mathrm{ddH}_{2} \mathrm{O}$ served as negative control.

\section{Sequence analysis and genetic characteri- zation of Cryptosporidium spp. isolates}

The PCR products with positive amplifications were purified and sequenced. Then, the obtained sequences were subjected to BLAST website (https:/ / blast.ncbi.nlm.nih.gov/Blast.cgi) to align with the reference sequences to determine the species and subtypes of Cryptosporidium spp. isolates. The novel nucleotide sequences were deposited in the GenBank database under the accession numbers MN704639-41 and MN747318-20.

\section{Statistical analysis}

Data analyses were performed using SPSS software (version 20.0, IBM, Armonk, NY, USA). The differences between different groups were assessed by Pearson chi-square $\left(\chi^{2}\right)$ or Fisher's exact test where indicated. All tests were two-sided, and $P<0.05$ was considered statistically significant.

\section{Results}

In total, Cryptosporidium spp. was detected in 26 out of 195 patients with diagnosed gastrointestinal cancers $(13.33 \%)$. Conversely, none of the control population has Cryptosporidium spp. infection (0/141; Table 1). The prevalence of Cryptosporidium spp. in gastrointestinal cancer patients was significantly higher than that in controls $\left(x^{2}=13.904, \mathrm{df}=1, P<0.001\right)$. In detail, the infection rates of Cryptosporidium spp. in colorectal, gastric, esophageal, liver and small intestine cancers were $17.24 \%(20 / 116), 4 \%(2 / 51)$, $6.25 \%(1 / 16), 14.29 \%(1 / 7)$ and $40 \% \quad(2 / 5)$, respectively (Table 1). Except for gastric cancer $(P=0.121)$, all the gastrointestinal cancers were significantly associated with Cryptosporidium spp. infections (Table 1).
Table 1. Frequencies of Cryptosporidium spp. in gastrointestinal cancer patients and controls

\begin{tabular}{lllll}
\hline Groups & No. examined & No. positive & $\%$ & $X^{2} / d f / P$-value \\
\hline Controls & 141 & 0 & 0 & Reference \\
Gastrointestinal cancers & 195 & 26 & 13.33 & $13.904 / 1 /<0.001$ \\
Cancer types & & & & \\
Colorectal cancer & 116 & 20 & 17.24 & $18.579 / 1 /<0.001$ \\
Gastric cancer & 51 & 2 & 4 & $-/-/ 0.121^{\#}$ \\
Esophageal cancer & 16 & 1 & 6.25 & $-/-/ 0.029^{\#}$ \\
Liver cancer & 7 & 1 & 14.29 & $15.054 / 1 /<0.001$ \\
Small intestinal cancer & 5 & 2 & 40 & $50 / 1 /<0.001$ \\
\hline \#Fisher's exact test. & & & &
\end{tabular}

Table 2. Frequencies of Cryptosporidium spp. in gastrointestinal cancer patients by age, sex and residence $(n=195)$

\begin{tabular}{|c|c|c|c|c|}
\hline Characteristic & No. examined & No. positive & $\%$ & $X^{2} / d f / P$-value \\
\hline \multicolumn{5}{|l|}{ Age (years) } \\
\hline$\leq 50$ & 44 & 6 & 13.64 & $0.058 / 2 / 0.972$ \\
\hline $51-60$ & 61 & 8 & 13.11 & \\
\hline$>60$ & 90 & 12 & 13.33 & \\
\hline \multicolumn{5}{|l|}{ Sex } \\
\hline Male & 125 & 20 & 16 & $2.24 / 1 / 0.134$ \\
\hline Female & 70 & 6 & 8.57 & \\
\hline \multicolumn{5}{|l|}{ Residence } \\
\hline Urban & 113 & 17 & 15.04 & $0.707 / 1 / 0.4$ \\
\hline Rural & 82 & 9 & 10.98 & \\
\hline
\end{tabular}

The age of gastrointestinal cancer patients ranged between 31-87 years with the average age of 59 years. Cryptosporidium spp. was found in all the age groups, but there was no obvious difference between different groups $(P=0.972$; Table 2$)$. The infection rate of Cryptosporidium spp. was nearly 2-fold higher in males than that in females ( $16 \%$ vs $8.57 \%$; Table 2 ). However, Since we collected nearly twice as many fecal samples from men as from women (125 vs. 70, Table S1), it is possible that the higher frequency of Cryptosporidium spp. infection in males than that in females was due to the larger sample size of males. Moreover, a slightly high frequency of Cryptosporidium spp. was observed in patients from urban areas (15.04\%) than that from rural areas (10.98\%; Table 2). However, no statistical significant difference was found between any of these groups. Similar tendencies of the infection rates were also observed in patients with colorectal cancer (Table S3). But the infection rate in males with colorectal cancer $(22.39 \%)$ was twice that in females $(10.2 \%)$, and this was statistical significance.

To determine the species for Cryptosporidium spp. infections in gastrointestinal cancer patients, nested PCR was used to detect the $18 S$ rRNA gene. The results indicated that all of the Cryptosporidium spp. identified were $C$. parvum. Of the 26 sequences obtained, 24 sequences were $100 \%$ identity to the reference sequences (GenBank ID: JX886768.1, JX886767.1, JX886766.1, JX886765.1, FJ752165.1, Table S4), while two sequences have single nucleotide polymorphisms (SNPs) and were assigned as novel types (Table 3 ). 
Table 3. SNPs of the $18 \mathrm{~S}$ rRNA gene of $C$. parvum identified in this study

\begin{tabular}{|c|c|c|c|c|c|c|c|c|c|}
\hline \multirow[t]{2}{*}{ Groups } & \multirow[t]{2}{*}{ GenBank ID } & \multirow[t]{2}{*}{ Isolate No. } & \multirow[t]{2}{*}{ Type } & \multicolumn{6}{|c|}{ SNPs } \\
\hline & & & & 369\# & $390^{\#}$ & $398^{\#}$ & $410^{\#}$ & $484^{\#}$ & $499 \neq$ \\
\hline \multirow[t]{3}{*}{ Colorectal cancer } & MN704639 & 18 & $\mathrm{CpCH}^{*}{ }^{*}$ & G & G & G & $\mathrm{C}$ & G & G \\
\hline & MN704640 & 1 & $\mathrm{CpCH} 2$ & G & G & G & G & G & G \\
\hline & MN704641 & 1 & $\mathrm{CpCH} 3$ & $\mathrm{~T}$ & A & $\mathrm{T}$ & G & $\mathrm{T}$ & A \\
\hline Gastric cancer & MN704639 & 2 & $\mathrm{CpCH} 1$ & G & G & G & $\mathrm{C}$ & G & G \\
\hline Esophageal cancer & MN704639 & 1 & $\mathrm{CpCH} 1$ & G & G & G & C & G & G \\
\hline Liver cancer & MN704639 & 1 & $\mathrm{CpCH} 1$ & G & G & G & $\mathrm{C}$ & G & G \\
\hline Small intestine cancer & MN704639 & 2 & $\mathrm{CpCH} 1$ & G & G & G & $\mathrm{C}$ & G & G \\
\hline
\end{tabular}

*CpCH: C. parvum Changchun Human.

"means the nucleotide position.

Table 4. Molecular characterization of $C$. parvum identified in this study

\begin{tabular}{|c|c|c|c|c|c|c|c|}
\hline GenBank ID & Type & Species & Genotype & Isolate no. & Cancer type (number of isolates) & Reference sequence & SNPs \\
\hline MN747318 & CPgp1 & C. parvum & IIaA15G2R1 & 12 & Colorectal (10), Gastric (1), Small intestine (1) & AY262034 & - \\
\hline MN747319 & CPgp2 & C. parvum & IIaA15G2R2 & 12 & Colorectal (9), Gastric (1), Small intestine (1), Esophageal (1) & DQ192501 & - \\
\hline MN747320 & CPgp3 & C. parvum & IIaA13G2R2 & 2 & Colorectal (1), Liver (1) & AY262034 & $\mathrm{T} 142 \mathrm{~A}$ \\
\hline
\end{tabular}

Although the prevalence of Cryptosporidium spp. has been documented in several digestive cancers, the subtypes have been rarely identified. Here, we used nested PCR amplifying gp60 gene to determine the subtypes of $C$. parvum in gastrointestinal cancer patients. Genetic analyses identified that of 26 isolates obtained, twelve were typed as IIaA15G2R1 and twelve were types as IIaA15G2R2 (Table 4). In addition, we found that another two sequences were homologous to IIaA15G2R1 subtype with the 142 position $\mathrm{T}$ substituted by $\mathrm{A}$. According to the nomenclature of Cryptosporidium spp., we defined this two novel sequences as IIaA13G2R2 (GenBank ID: MN747320) [24]. IIaA13G2R2 was only observed in colorectal and liver cancers, whereas IIaA15G2R1 and IIaA15G2R2 were found in colorectal, gastric and small intestine cancers. In addition, IIaA15G2R2 subtype was also observed in esophageal cancer. These results further confirmed that $C$. paroum was responsible for Cryptosporidium spp. infections in all gastrointestinal cancer patients, and suggested the potential zoonotic transmission.

\section{Discussion}

Cryptosporidium spp. infection is a severe threat to human health. It is one of the major causes of diarrhea and can be life-threatening among immunocompromised patients. To our knowledge, the present study is the first report performed in China comparing the prevalence of Cryptosporidium spp. infections between gastrointestinal cancer patients and people without digestive symptoms. The results herein indicated that Cryptosporidium spp. was strongly associated with gastrointestinal cancers, and C. paroum was responsible for the infections in digestive cancer patients. Except gastric cancer, all the studied cancer types showed statistically significant correlation with C. paroum infection, indicating that it could be a potential etiological factor for these cancers.

The associations of Cryptosporidium spp. Infections with digestive cancers have been described in different populations. Several epidemiological studies demonstrated that colorectal cancer is highly correlated with Cryptosporidium spp. [10-14]. In consistence, we here found that $17.24 \%$ patients with colorectal cancer had C. parvum infections, which was significantly higher than control population. This infection rate was similar with the previous reports in colorectal cancer patients: $18 \%, 12.6 \%$ and $13 \%$ in Poland, and $21 \%$ in Lebanon. A link has also been established between cryptosporidiosis and liver cancer $[14,15,25]$. This was also true in our study which showed that $C$. paroum infection was significantly higher in liver cancer patients than that in control population. In addition, we observed 2 out of 51 patients with gastric cancer had C. parvum. However, statistical analysis did not reveal the significant correlation between gastric cancer and $C$. parvum infection. This is consistent with the previous study among Lebanese which found none of the gastric cancer patients had Cryptosporidium spp. infection [13]. Furthermore, for the first time, our study identified the high occurrences of $C$. paroum in patients with esophageal cancer $(6.25 \%, 1 / 16)$ and small intestine cancer $(40 \%, 2 / 5)$. However, due to the small sample size, further studies with a large collection of samples and in different populations are needed to reach the definitive conclusions of the correlation between Cryptosporidium spp. infection and these two cancers.

In fact, the ability of Cryptosporidium spp. in the induction of digestive malignancies has been demonstrated in rodent models. Several studies 
showed that animal-derived C. parvum can induce the generation of colorectal, stomach and small intestine tumors using dexamethasone treated SCID (severe combined immunodeficiency) mice [26-29]. Also, C. parvum of human origin can cause gastrointestinal and biliary adenocarcinoma in SCID mice [30]. In contrast, another species $C$. muris did not induce the gastrointestinal cell transformation in the same experimental model [27], suggesting the unique role of C. parvum in the development of digestive cancers.

Till now, more than 30 species of Cryptosporidium have been identified. C. paroum and C. hominis account for most of Cryptosporidium spp. infections in humans, while C. meleagridis, C. felis, C. canis, C. muris, C. suis and $C$. andersoni are occasionally observed in humans $[7,31]$. Previous molecular analyses indicated that $C$. hominis and $C$. paroum species were responsible for Cryptosporidium infections in colorectal cancer patients [13]. In the present study, we found that all the Cryptosporidium spp. identified in gastrointestinal cancer patients were C. paroum. C. parvum is one of the major zoonotic species with a wide range of hosts including humans, cattle and horses [7]. BLAST analyses indicated that most of the $18 S$ rRNA sequences obtained were $100 \%$ identity with the GenBank reference sequences: JX886768.1, JX886767.1, JX886766.1, JX886765.1 and FJ752165.1 (Table S4), which were all isolated from cattle, suggesting the potential zoonotic transmission. In addition, two novel sequences were obtained which displayed SNPs when compared with the above reference sequences (Table 3).

Previously, IlaA15G2R1 (one case) and IIcA5G3 (two cases) subtypes in colorectal cancer, and IIaA16G2R1 (one case) and IIaA17G2R1 (22 cases) subtypes in paediatric oncology have been identified $[5,23,32]$. However, the subtypes responsible for $C$. paroum infections in other gastrointestinal cancers have not been determined yet. We here identified that IIaA15G2R1, IIaA15G2R2 and IIaA13G2R2 were the subtypes responsible for $C$. parvum infections in gastrointestinal cancers. Noticeably, two novel sequences with 13 TCA (A), 2 TCG (G) and 2 ACA TCA $(R)$ repeats were first identified and defined as IIaA13G2R2 subtype and were observed in both colorectal and liver cancers. For the prevalence of subtypes in colorectal cancer, IIaA15G2R1 and IIaA15 G2R2 were the predominant subtypes and were detected in almost equal frequencies, whereas IIaA13 G2R2 was only found in one case. Additionally, both IIaA15G2R1 and IIaA15G2R2 were observed in small intestine cancer, but only IIaA15G2R2 has been found in esophageal cancer. It has been demonstrated that subtype families of $C$. paroum have different host specificity. Ila family is mainly found in cattle and is responsible for zoonotic cryptosporidiosis [24]. IIaA15 G2R1 is the most prevalent subtype for C. parvum worldwide and is mostly found in calves [7], while IIaA15G2R2 has been identified in calves and humans in the United States [33]. Thus, these results further supported a potential zoonotic transmission of cryptosporidiosis in digestive cancer patients. Nevertheless, the transmission sources and hosts of IIaA13G2R2 subtype need to be further investigated.

The pathological mechanism underlying Cryptosporidium spp. infections in the induction of digestive cancers remains unclear. However, available data suggested that Cryptosporidium spp., for its survival and transmission, can interfere with the cell signaling pathways and impact the gene expressions in host cells, thus, may secondarily promote the generation of gastrointestinal cancers. It has been shown that $C$. parvum can inhibit the apoptosis of infected biliary epithelia through activating the NF-kB signaling pathway [34]. A microarray analysis also revealed that $C$. parvum infection affected a large number of apoptosis gene expressions with upregulation of antiapoptotic genes and downregulation of proapoptotic genes at early stage [35]. Resistance to apoptosis is one of the hallmarks of cancer development [36]. Thus, C. paroum may induce the gastrointestinal cancers via preventing infected cells from cell death. Additionally, Benamrouz S. et al. found that $C$. parvum infections can alter the Wnt signaling pathway components and is able to modulate the cytoskeleton network in infected cells. They also showed that the changes of these biological processes were involved in the progression of ileo-caecal oncogenesis [37]. However, further work is still needed to clarify the molecular mechanisms of gastrointestinal epithelia transformation induced by Cryptosporidium.

In conclusion, the present study, for the first time, estimated the prevalence of Cryptosporidium spp. and characterized its species and subtypes in gastrointestinal cancer patients in China. Nevertheless, the causal relationship of C. parvum infections in the development of gastrointestinal cancers needs to be further elucidated.

\section{Abbreviations}

AIDS: Acquired Immune Deficiency Syndrome; CT: computed tomography; NMR: nuclear magnetic resonance; SCID: severe combined immunodeficiency; SNPs: single nucleotide polymorphisms.

\section{Supplementary Material}

Supplementary tables.

http://www.jcancer.org/v11p3334s1.pdf 


\section{Acknowledgments}

We thank Zhanjie Cong and Chunying Tian (Jilin Cancer Hospital) for assistance of the fecal specimen collection.

\section{Funding}

This work was supported by the grants from the National Key Research and Development Program of China (No. 2017YFD0501305), National Natural Science Foundation of China (Nos. 31972704, 31872465), Science and Technology Development Program of Jilin Province (No. 20190103075JH) and China Postdoctoral Science Foundation (No. 2019M651196).

\section{Competing Interests}

The authors have declared that no competing interest exists.

\section{References}

1. Bray F, Ferlay J, Soerjomataram I, et al Global cancer statistics 2018: GLOBOCAN estimates of incidence and mortality worldwide for 36 cancers in 185 countries. CA Cancer J Clin. 2018; 68: 394-424.

2. Ferlay J, Colombet M, Soerjomataram I, et al. Estimating the global cancer incidence and mortality in 2018: GLOBOCAN sources and methods. Int J Cancer. 2019; 144: 1941-53.

3. zur Hausen $H$. The search for infectious causes of human cancers: where and why (Nobel lecture). Angew Chem Int Ed Engl. 2009; 48: 5798-808.

4. de Martel C, Franceschi S. Infections and cancer: established associations and new hypotheses. Crit Rev Oncol Hematol. 2009; 70: 183-94.

5. Kalantari N, Gorgani-Firouzjaee T, Ghaffari S, et al. Association between Cryptosporidium infection and cancer: A systematic review and meta-analysis. Parasitol Int. 2020; 74: 101979.

6. Ryan U, Zahedi A, Paparini A. Cryptosporidium in humans and animals-a one health approach to prophylaxis. Parasite Immunol. 2016; 38: 535-47.

7. Feng Y, Ryan UM, Xiao L. Genetic Diversity and Population Structure of Cryptosporidium. Trends Parasitol. 2018; 34: 997-1011.

8. Koehler AV, Korhonen PK, Hall RS, et al. Use of a bioinformatic-assisted primer design strategy to establish a new nested PCR-based method for Cryptosporidium. Parasit Vectors. 2017; 10: 509.

9. Izquierdo J, Antunez I, Calderon MT, et al. Diarrhea caused by Cryptosporidium and colonic neoplasia. Rev Clin Esp. 1988; 182: 393-4.

10. Sulzyc-Bielicka V, Kuzna-Grygiel W, Kolodziejczyk L, et al. Cryptosporidiosis in patients with colorectal cancer. J Parasitol. 2007; 93: 722-4.

11. Sulzyc-Bielicka V, Kolodziejczyk L, Jaczewska S, et al. Prevalence of Cryptosporidium sp. in patients with colorectal cancer. Pol Przegl Chir. 2012; 84: 348-51.

12. Sulzyc-Bielicka V, Kolodziejczyk L, Jaczewska S, et al. Colorectal cancer and Cryptosporidium spp. infection. PLoS One. 2018; 13: e0195834.

13. Osman M, Benamrouz S, Guyot K, et al. High association of Cryptosporidium spp. infection with colon adenocarcinoma in Lebanese patients. PLoS One. 2017; 12: e0189422.

14. Sanad MM, Thagfan FA, Al Olayan EM, et al. Opportunistic Coccidian Parasites among Saudi Cancer Patients Presenting with Diarrhea: Prevalence and Immune Status. Res J Parasitol. 2014; 9: 55-63.

15. Mousa N, Abdel-Razik A, El-Nahas H, et al. Cryptosporidiosis in patients with diarrhea and chronic liver diseases. J Infect Dev Ctries. 2014; 8: 1584-90.

16. Liu H, Shen Y, Yin J, et al. Prevalence and genetic characterization of Cryptosporidium, Enterocytozoon, Giardia and Cyclospora in diarrheal outpatients in China. BMC Infect Dis. 2014; 14: 25.

17. Tian LG, Chen JX, Wang TP, et al. Co-infection of HIV and intestinal parasites in rural area of China. Parasit Vectors. 2012; 5: 36.

18. Feng Y, Wang L, Duan L, et al. Extended outbreak of cryptosporidiosis in a pediatric hospital, China. Emerg Infect Dis. 2012; 18: 312-4.

19. Wang RJ, Li JQ, Chen YC, et al. Widespread occurrence of Cryptosporidium infections in patients with HIV/AIDS: Epidemiology, clinical feature, diagnosis, and therapy. Acta Trop. 2018; 187: 257-63.

20. Yang D, Yang Y, Wang Y, et al. Prevalence and Risk Factors of Ascaris lumbricoides, Trichuris trichiura and Cryptosporidium Infections in Elementary School Children in Southwestern China: A School-Based Cross-Sectional Study. Int J Environ Res Public Health. 2018; 15: 1809.

21. Zhang N, Zhang H, Yu Y, et al. High prevalence of Pentatrichomonas hominis infection in gastrointestinal cancer patients. Parasit Vectors. 2019; 12: 423.
22. Ryan U, Xiao L, Read C, et al. Identification of novel Cryptosporidium genotypes from the Czech Republic. Appl Environ Microbiol. 2003; 69: 4302-7.

23. Hijjawi N, Zahedi A, Kazaleh M, et al. Prevalence of Cryptosporidium species and subtypes in paediatric oncology and non-oncology patients with diarrhoea in Jordan. Infect Genet Evol. 2017; 55: 127-30.

24. Xiao L, Feng Y. Molecular epidemiologic tools for waterborne pathogens Cryptosporidium spp. and Giardia duodenalis. Food and Waterborne Parasitology. 2017; 8-9: 14-32

25. Tomizawa D, Imai K, Ito S, et al. Allogeneic hematopoietic stem cell transplantation for seven children with X-linked hyper-IgM syndrome: a single center experience. Am J Hematol. 2004; 76: 33-9.

26. Certad G, Ngouanesavanh T, Guyot K, et al. Cryptosporidium parvum, a potential cause of colic adenocarcinoma. Infect Agent Cancer. 2007; 2: 22.

27. Certad G, Creusy C, Ngouanesavanh T, et al. Development of Cryptosporidium parvum-induced gastrointestinal neoplasia in severe combined immunodeficiency (SCID) mice: severity of lesions is correlated with infection intensity. Am J Trop Med Hyg. 2010; 82: 257-65.

28. Certad G, Creusy C, Guyot K, et al. Fulminant cryptosporidiosis associated with digestive adenocarcinoma in SCID mice infected with Cryptosporidium parvum TUM1 strain. Int J Parasitol. 2010; 40: 1469-75.

29. Benamrouz S, Guyot K, Gazzola S, et al. Cryptosporidium parvum infection in SCID mice infected with only one oocyst: qPCR assessment of parasite replication in tissues and development of digestive cancer. PLoS One. 2012; 7: e51232.

30. Certad G, Benamrouz S, Guyot K, et al. Fulminant cryptosporidiosis after near-drowning: a human Cryptosporidium parvum strain implicated in invasive gastrointestinal adenocarcinoma and cholangiocarcinoma in an experimental model. Appl Environ Microbiol. 2012; 78: 1746-51.

31. Xiao L. Molecular epidemiology of cryptosporidiosis: an update. Exp Parasitol. 2010; 124: 80-9.

32. Essid R, Menotti J, Hanen C, et al. Genetic diversity of Cryptosporidium isolates from human populations in an urban area of Northern Tunisia. Infect Genet Evol. 2018; 58: 237-42.

33. Xiao L, Zhou L, Santin M, et al. Distribution of Cryptosporidium parvum subtypes in calves in eastern United States. Parasitol Res. 2007; 100: 701-6.

34. Chen XM, Levine SA, Splinter PL, et al. Cryptosporidium parvum activates nuclear factor kappaB in biliary epithelia preventing epithelial cell apoptosis. Gastroenterology. 2001; 120: 1774-83.

35. Liu J, Deng M, Lancto CA, et al. Biphasic modulation of apoptotic pathways in Cryptosporidium parvum-infected human intestinal epithelial cells. Infect Immun. 2009; 77: 837-49.

36. Hanahan D, Weinberg RA. Hallmarks of cancer: the next generation. Cell. 2011; 144: 646-74.

37. Benamrouz S, Conseil V, Chabe M, et al. Cryptosporidium parvum-induced ileo-caecal adenocarcinoma and Wnt signaling in a mouse model. Dis Model Mech. 2014; 7: 693-700. 